Supporting Information for

\title{
Heterobilayer with ferroelectric switching of topological state
}

\author{
Jun-Jie Zhang ${ }^{1}$, Dongyang Zhu' ${ }^{2}$, and Boris Yakobson ${ }^{1 *}$ \\ ${ }^{1}$ Department of Materials Science and NanoEngineering, Rice University, Houston, Texas \\ 77005, USA \\ ${ }^{2}$ Department of Chemical and Biomolecular Engineering, Rice University, Houston, Texas \\ 77005, USA \\ Email: biy@rice.edu
}

\section{More computational details}

All calculations were performed using the plane wave basis Vienna $a b$ initio simulation package (VASP) code, ${ }^{1-2}$ the generalized gradient approximation in the Perdew-BurkeErnzerhof (GGA-PBE) formulation is used, with a cutoff energy of $400 \mathrm{eV}$. A vacuum space of $25 \AA$ minimizes the interactions of neighboring slabs, and 2D Brillouin zone is sampled with $24 \times 24 k$-points. Our calculations included the out-plane dipole correction. Due to the weak $\mathrm{vdW}$ force in heterobilayer, several kinds of vdW corrections (DFT-D2, DFT-D3, and optB86b-vdW) and more accurate SCAN (strongly constrained and appropriately normed) with rVV10 functional were employed. ${ }^{2-5}$ The topological properties were characterized by the calculations with Wannier functions, as implemented in Wannier90 package. ${ }^{6}$ To construct the 
Wannier functions, the Bloch states of $\mathrm{Sb} p, \mathrm{Se} s, p$ and $\operatorname{In} p$ orbitals are projected from DFT band structures.

a
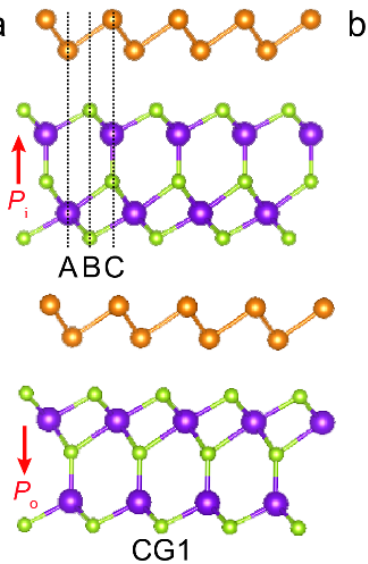

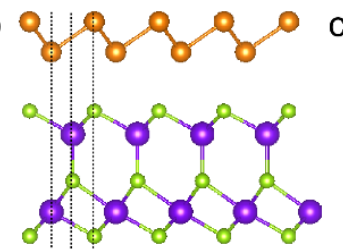

$A B C$
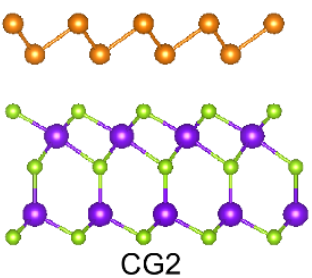
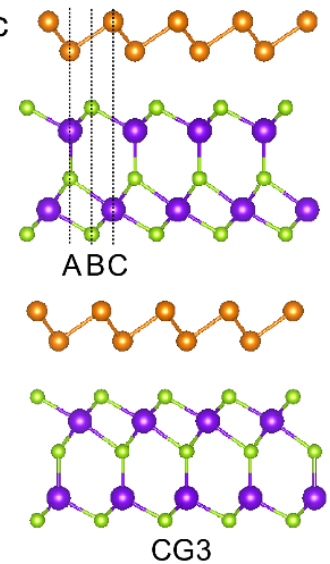

Figure S1. Side view of three most possible stacking constructions (CG) for $\beta$-Sb/ $/ n_{2} \mathrm{Se}_{3}$ vertical heterostructure. Upper layer for $P_{\mathrm{i}}$, down layer for $P_{\mathrm{o}}$.

Table S1. Calculated total energies (meV) of three stacking constructions using different van der Waals corrections, as DFT-D2, DFT-D3, DFT-DF (optB88) and SCAN-rVV10. The energies of CG1 are taken as reference.

\begin{tabular}{ccccc}
\hline \hline$P_{\mathrm{i}}$ & D2 & D3 & DF-optB88 & SCAN-rVV10 \\
\hline CG1 & 0 & 0 & 0 & 0 \\
CG2 & -6.52 & 2.00 & 4.47 & 7.16 \\
CG3 & 3.11 & 9.76 & 15.23 & 38.67 \\
\hline$P_{\mathrm{o}}$ & $\mathrm{D} 2$ & $\mathrm{D} 3$ & DF-optB88 & SCAN-rVV10 \\
\hline CG1 & 0 & 0 & 0 & 0 \\
CG2 & -1.61 & 22.75 & 25.48 & 0.24 \\
CG3 & 13.15 & 37.68 & 40.99 & 20.76 \\
\hline \hline
\end{tabular}




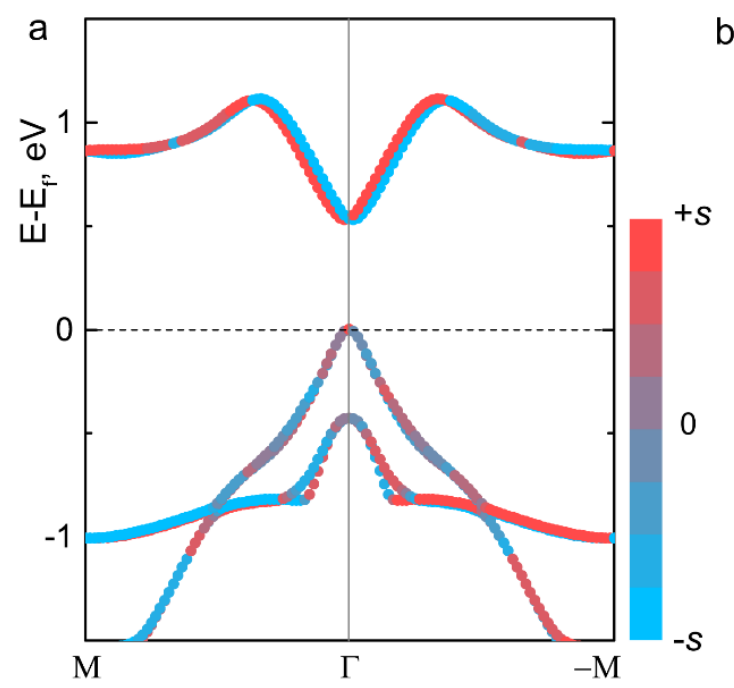

b
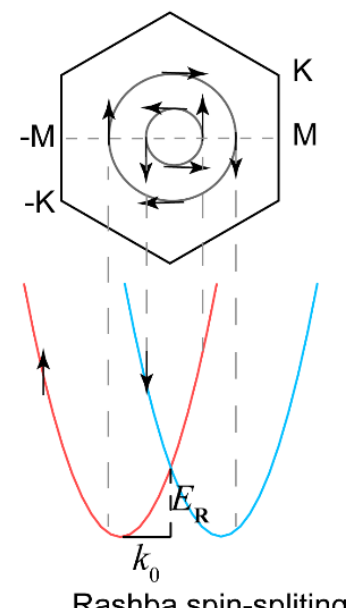

Rashba spin-spliting

Figure S2. (a) Spin-decomposed band structure of $P_{\mathrm{i}}$ with SOC effects. (b) Upper: $+S(-S)$ is decomposed along the $\mathrm{M}$ to $\mathrm{K}$ (-M to $-\mathrm{K})$ direction. Down: sketch of Rashba spin-spliting with opposite spin dispersion.
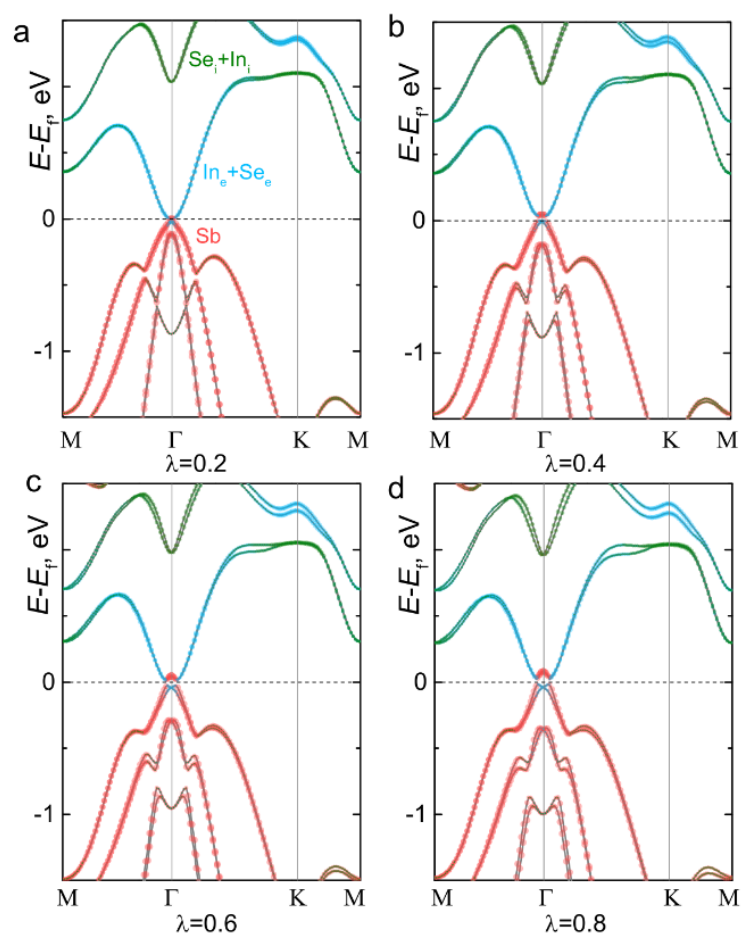

Figure S3. Band structure of $P_{\mathrm{i}}$ with various SOC strength $\lambda$, from 0.2 to 0.8 . 


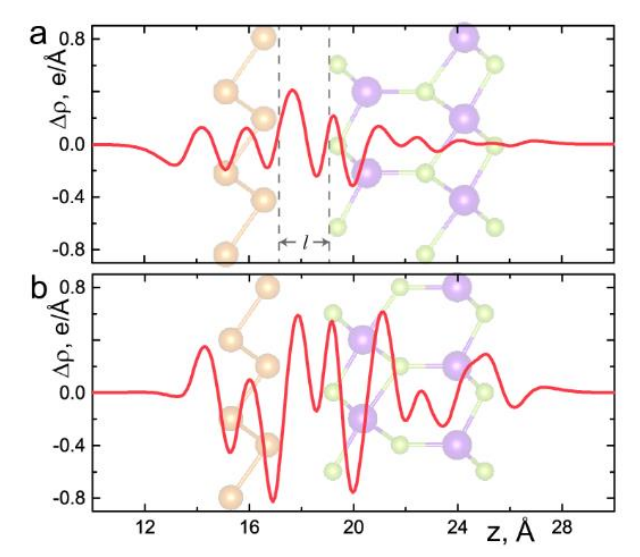

Figure S4. Integrals of differential charge densities of $\beta$-Sb/ $\operatorname{In}_{2} \mathrm{Se}_{3}$ heterostructure along $z$ direction. (a) $P_{\mathrm{i}}$ structure; (b) $P_{\mathrm{o}}$ structure.

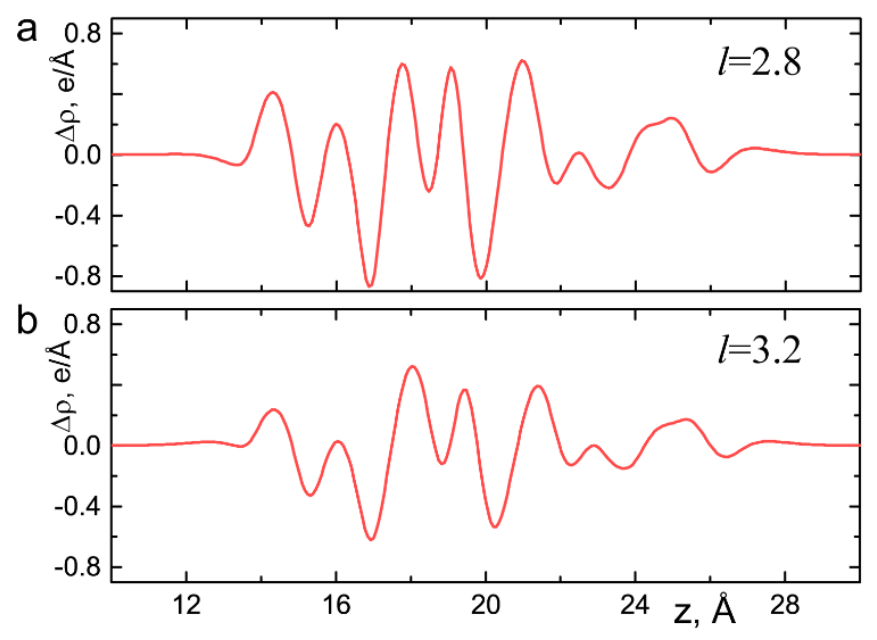

Figure S5. Integrals of differential charge densities of $P_{\mathrm{o}}$ along $z$ direction. The distances between $\beta$-Sb and $\operatorname{In}_{2} \mathrm{Se}_{3}$ layer $l$ are fixed to 2.8 and $3.2 \AA$, respectively.
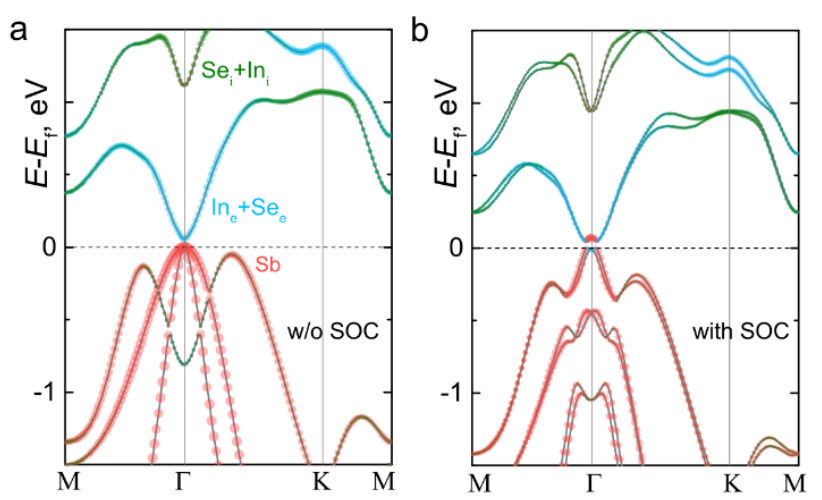

Figure S6. Band structures of $P_{\mathrm{o}}$ (a) without and (b) with SOC effects. The $l$ is fixed to $2.7 \AA$ $\left(\left(\Delta l / l_{0}=-7 \%\right)\right.$. 


\section{Tight-Binding Model}

The tight-binding (TB) model is used to explain the band inversion around $\Gamma$ point. In the conduction band minimum $(\mathrm{CBM}), \mathrm{In}_{\mathrm{i}} / \mathrm{In}_{\mathrm{o}}-s$ orbitals have more contributions than $\mathrm{Se}_{\mathrm{i}} / \mathrm{Se}_{\mathrm{o}^{-}}-p z$ orbitals, so $\mathrm{Se}_{\mathrm{i}} / \mathrm{Se}_{\mathrm{o}}-p z$ orbitals are not considered in our TB model. A minimal basis of three orbitals $\left(\mathrm{In}_{\mathrm{i}} / \mathrm{In}_{\mathrm{o}}-S, \mathrm{Sb}-p x\right.$ and $\left.\mathrm{Sb}-p y\right)$ is employed to construct the TB model. To simplify, Rashba SOC is ignored here since QSH is not determined by Rashba energies splitting. The total spin-up Hamiltonian is written in the basis $\left(\operatorname{In}_{\mathrm{i}} / \mathrm{In}_{\mathrm{o}}-S, \mathrm{Sb}-p x\right.$ and $\left.\mathrm{Sb}-p y\right)$ as,

$$
H=H_{0}+H_{S O C}=\left(\begin{array}{ccc}
h_{s s} & h_{s x} & h_{s y} \\
h_{x s}^{*} & h_{x x} & h_{x y} \\
h_{y s}^{*} & h_{y x}^{*} & h_{y y}
\end{array}\right)+\left(\begin{array}{ccc}
0 & 0 & 0 \\
0 & 0 & -i \lambda_{S O} \\
0 & i \lambda_{S O} & 0
\end{array}\right)
$$

in which

$$
\begin{aligned}
& h_{s s}=\varepsilon_{s}+2 V_{s s \sigma}(2 \cos \zeta \cos v+\cos 2 \zeta) \\
& h_{s x}=2 i V_{s p \sigma}(\sin \zeta \cos v+\sin 2 \zeta) \\
& h_{s y}=2 \sqrt{3} i V_{s p \sigma} \cos \zeta \sin v \\
& h_{x x}=\varepsilon_{P}+\left(V_{p p \sigma}+3 V_{p p \pi}\right) \cos \zeta \cos v+2 V_{p p \sigma} \cos 2 \zeta \\
& h_{x y}=-\sqrt{3}\left(V_{p p \sigma}-V_{p p \pi}\right) \sin \zeta \cos v \\
& h_{y y}=\varepsilon_{P}+\left(V_{p p \pi}+3 V_{p p \sigma}\right) \cos \zeta \cos v+2 V_{p p \pi} \cos 2 \zeta \\
& (\zeta, v)=\left(\frac{1}{2} a k_{x}, \frac{\sqrt{3}}{2} a k_{y}\right)
\end{aligned}
$$

where $\varepsilon_{s}$ and $\varepsilon_{p}$ are on-site energies for $s$ and $p_{x}, p_{y}$ orbitals, respectively. $V_{s s \sigma}, V_{s p \sigma}$ and $V_{p p \sigma}$ are $\sigma$ type hopping energies; $V_{p p \pi}$ is $\pi$ type hopping energy; $\lambda_{\mathrm{R}}$ is the strength of SOC. In and $\mathrm{Sb}$ atoms occupy two different triangular lattice planes, and the coupling between them is 
negligible as we found. Thus, we can reasonably ignore the hopping between the In-s and Sb$p x, p y$ orbitals along the $z$ direction. The on-site energies and hopping energies are calculated through Wannier function (Table S2), which are fitting well with DFT bands around $\Gamma$ point (Figure R2).

Table S2. The obtained on-site energies and hopping energies based on Wannier function.

\begin{tabular}{lllllll}
\hline \hline & $\varepsilon_{\mathrm{s}}$ & $\varepsilon_{\mathrm{p}}$ & $V_{\mathrm{ss} \sigma}$ & $V_{\mathrm{sp} \sigma}$ & $V_{\mathrm{pp} \sigma}$ & $V_{\mathrm{pp} \pi}$ \\
\hline $\mathrm{E}(\mathrm{eV})$ & $2.85\left(\mathrm{In}_{\mathrm{o}}\right) / 3.67\left(\mathrm{In}_{\mathrm{i}}\right)$ & -3.54 & -0.48 & 0.01 & 1.10 & 0.08 \\
\hline \hline
\end{tabular}

Without the consideration of SOC, we obtained a nondegenerate eigenvalue $E_{s}=\varepsilon_{s}+6 V_{s s \sigma}$ and two degenerate eigenvalues $E_{p_{x}, p_{y}}=\varepsilon_{P}+\left(3 V_{p p \pi}+3 V_{p p \sigma}\right)$ at $\Gamma$ point. When SOC is considered, the eigenvalues at $\Gamma$ point change as follows,

$$
\begin{aligned}
& E_{s}^{\prime}=\varepsilon_{s}+6 V_{s s \sigma} \\
& E_{m_{j}=3 / 2}=\varepsilon_{P}+\left(3 V_{p p \pi}+3 V_{p p \sigma}\right)+\lambda_{s O} \\
& E_{m_{j}=1 / 2}=\varepsilon_{P}+\left(3 V_{p p \pi}+3 V_{p p \sigma}\right)-\lambda_{S O} .
\end{aligned}
$$

Among these orbitals, $\mathrm{In}_{\mathrm{i}} / \mathrm{In}_{\mathrm{o}}-S$ orbital and $\mathrm{Sb}-m_{j}=3 / 2$ state occupy around Fermi level, while $\mathrm{Sb}-m_{j}=1 / 2$ state has lower energy. In Table $\mathrm{S} 2$, the onsite energy of $\operatorname{In}_{\mathrm{o}}-S$ orbital is smaller than $\mathrm{In}_{\mathrm{i}}-S$ orbital due to the band offset caused by ferroelectric $\operatorname{In}_{2} \mathrm{Se}_{3}$ layer. In the outwardpolarization of $\operatorname{In}_{2} \mathrm{Se}_{3}$, the energy of $\mathrm{In}_{0}-S$ and $\mathrm{Sb}$ - $p x$, py orbitals around $\Gamma$ point are very close (Figure S7a), which enables the SOC to cause the band inversion between these two states. The corresponding re-opened band gap at $\Gamma$ point is $\varepsilon_{P}+\left(3 V_{p p \pi}+3 V_{p p \sigma}\right)+\lambda_{S O}-\left(\varepsilon_{s}+6 V_{s s \sigma}\right)$ (Figure S7b). However, the large energy difference between $\operatorname{In}_{\mathrm{i}}-S$ and $S b-p_{x}, p_{y}$ orbitals due to 
potential offset will prevent the band inversion in the inward-polarization caused by the relatively weak SOC (Figure S7c and S7d).

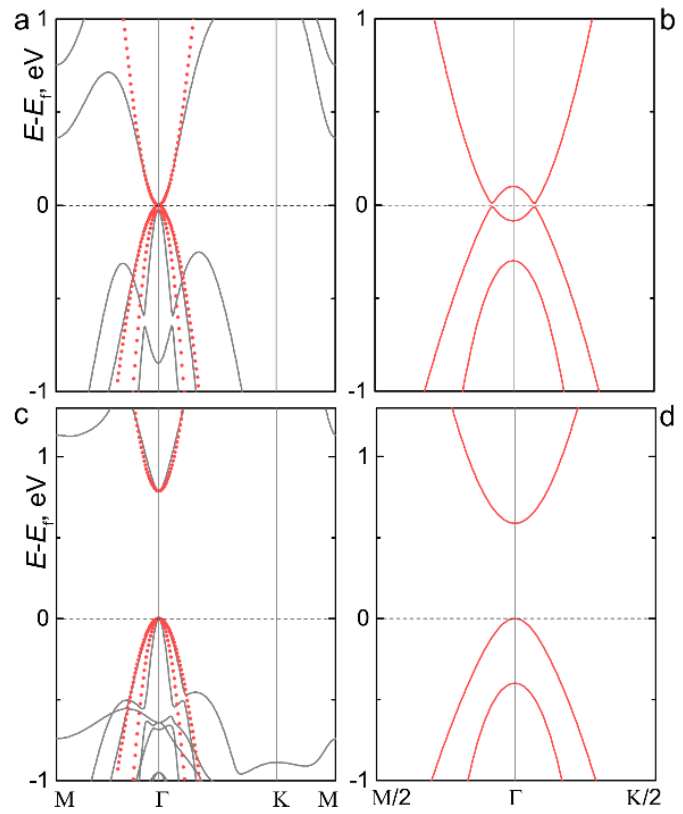

Figure S7. TB model band structure of $\beta-\mathrm{Sb} / \operatorname{In}_{2} \mathrm{Se}_{3}$ heterobilayer around $\Gamma$ point. (a-b) are for $P_{\mathrm{o}}$ outward-polarization, (a) is $\lambda_{\mathrm{SO}}=0$ and (b) is $\lambda_{\mathrm{SO}}=0.2 \mathrm{eV}$. (c-e) are for $P_{\mathrm{i}}$ inward-polarization, (c) is $\lambda_{\mathrm{SO}}=0$ and (d) is $\lambda_{\mathrm{SO}}=0.2 \mathrm{eV}$. Red marks the TB model bands; Grey marks DFT bands. The onsite and hopping energies used in TB model are listed in Table S2.
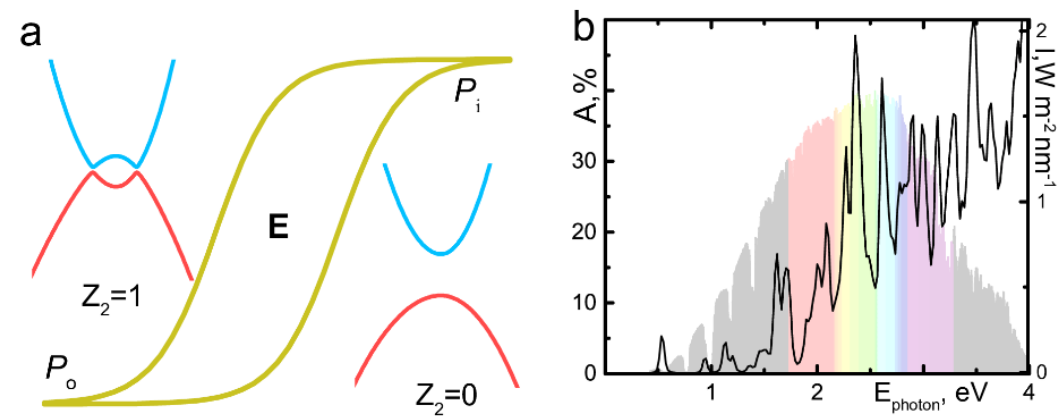

Figure S8. (a) Schematic hysteresis loop for the $P$ of $\operatorname{In}_{2} \mathrm{Se}_{3}$ layer under an external electric field, on the left side switching the heterobilayer into topologically nontrivial state $\left(Z_{2}=1\right)$ while on the right side in trivial, semiconductor $\left(\mathbb{Z}_{2}=0\right)$. (b) The calculated optical absorbance spectra 
of inward-polarized $\operatorname{In}_{2} \mathrm{Se}_{3}$ heterobilyer. The reference air-mass 1.5-solar spectral irradiance (I) is plotted in different colors from red to purple, indicating the visible light spectrum.
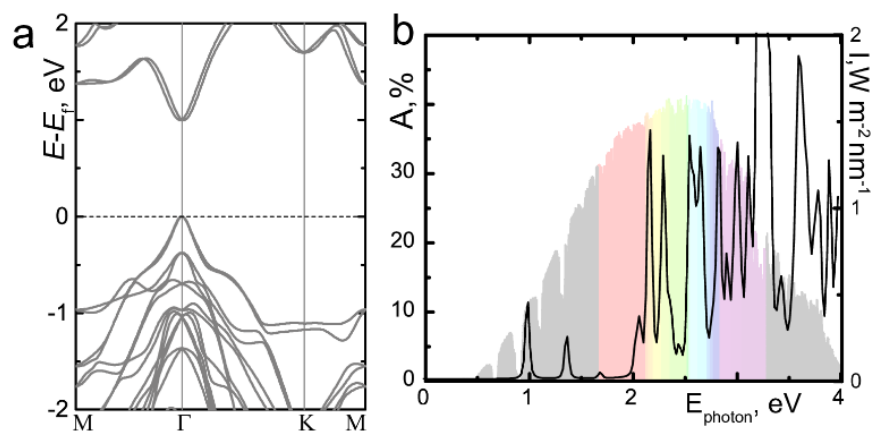

Figure S9. (a) Band structure of inward-polarization calculated by HSE06 functional plus SOC.

(b) The optical absorbance spectra of inward-polarized $\operatorname{In}_{2} \mathrm{Se}_{3}$ heterobilyer HSE06 functional plus SOC.
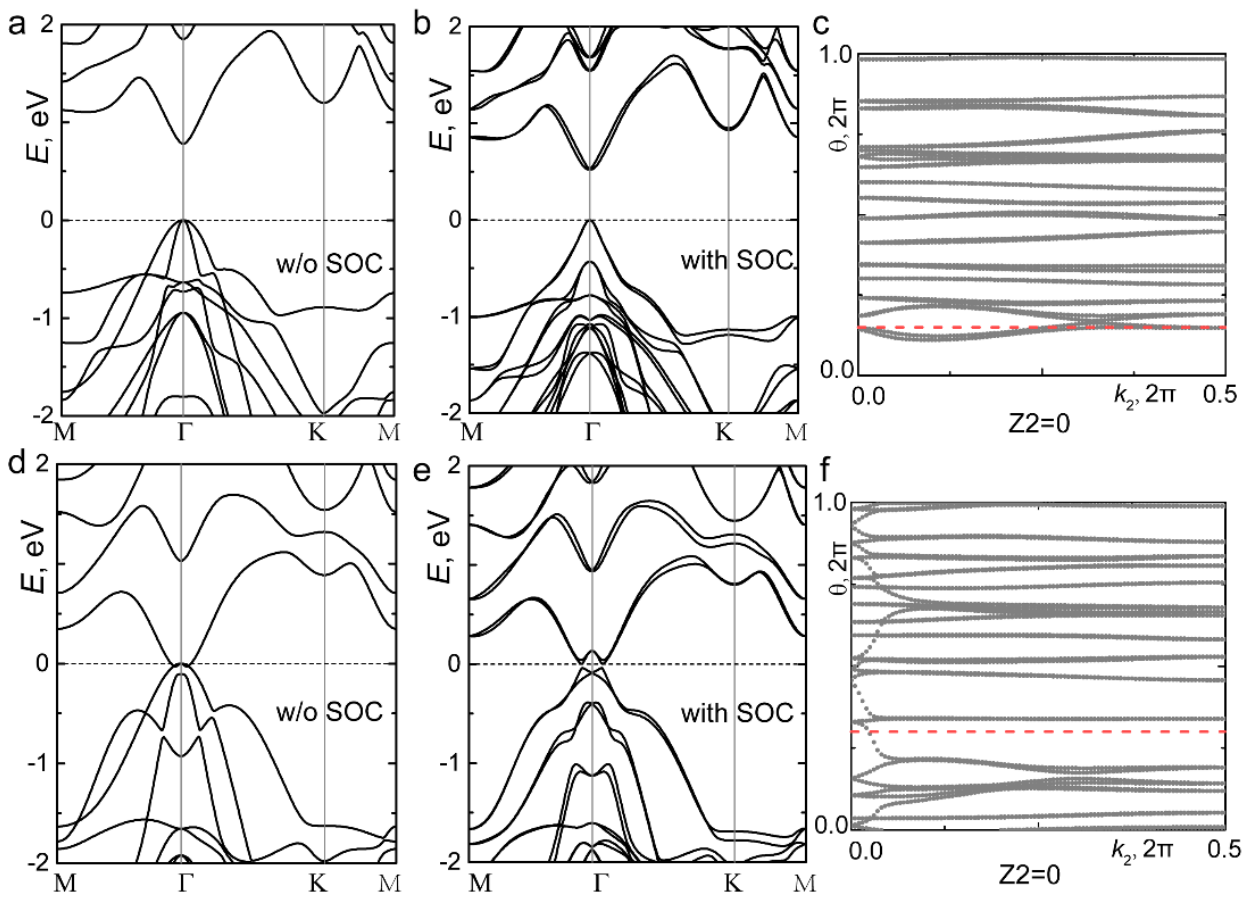

Figure S10. Band structures and evolution of the Wannier charge centers (WCCs) along $k_{\mathrm{y}}$ of

CG2 stacking. Upper layer for $P_{\mathrm{i}}$, low layer for $P_{\mathrm{o}}$. 


\section{REFERENCES}

1. Kresse, G.; Furthmüller, J., Efficient iterative schemes for ab initio total-energy calculations using a plane-wave basis set. Phys. Rev. B 1996, 54 (16), 11169.

2. Grimme, S., Semiempirical GGA-type density functional constructed with a long-range dispersion correction. J. Comput. Chem. 2006, 27 (15), 1787-1799.

3. Grimme, S.; Antony, J.; Ehrlich, S.; Krieg, H., A consistent and accurate ab initio parametrization of density functional dispersion correction (DFT-D) for the 94 elements $\mathrm{H}-\mathrm{Pu}$. J. Chem. Phys. 2010, 132 (15), 154104.

4. Klimeš, J.; Bowler, D. R.; Michaelides, A., Chemical accuracy for the van der Waals density functional. J. Phys.: Condens. Matter 2009, 22 (2), 022201.

5. Peng, H.; Yang, Z.-H.; Perdew, J. P.; Sun, J., Versatile van der Waals density functional based on a meta-generalized gradient approximation. Phys. Rev. X 2016, 6 (4), 041005.

6. Mostofi, A. A.; Yates, J. R.; Lee, Y.-S.; Souza, I.; Vanderbilt, D.; Marzari, N., wannier90: A tool for obtaining maximally-localised Wannier functions. Comput. Phys. Commun. 2008, $178(9), 685-699$. 